\title{
21
}

\section{GIS Applications for Water Distribution Systems}

Uzair (Sam) Shamsi

This chapter describes the geographic information system (GIS) applications for water distribution systems. The GIS applications that are covered include, development of hydraulic models, creation of thematic maps of the model output results, network simplification (skeletonization) for hydraulic modeling, estimation of node demands, estimation of node elevations, water main isolation (i.e., identifying the valves to be closed for repairing or replacing a broken water main), and delineation of pressure zones.

\subsection{Introduction}

More than $80 \%$ of all information used by a water utility is geographically referenced (Shamsi, 2002). While GIS applications for water distribution systems are not new, getting beyond the basic inventory and mapping functions is often challenging. Unless a water system GIS is taken to the operational level, it's just a pretty map. That is why the GIS emphasis is now shifting from computerized mapping to enterprise-wide mission critical applications. GIS applications make the routine business functions easier to perform which improves productivity and reduces cost. GIS applications have the potential to prepare our water systems for the operational challenges of the twenty-first century. This chapter describes seven useful GIS applications for typical hydraulic modeling and system operation tasks in a water distribution system. The case studies presented in the chapter illustrate that many water utilities are

Shamsi, U.M. 2004. "GIS Applications for Water Distribution Systems." Journal of Water

Management Modeling R220-21. doi: 10.14796/JWMM.R220-21.

(c) CHI 2004 www.chijournal.org ISSN: 2292-6062 (Formerly in Innovative Modeling of

Urban Water Systems. ISBN: 0-9683681-9-0) 
successfully using the GIS applications for modeling and operation of their water distribution systems. Various software examples presented in the chapter indicate that commercial-off-the-shelf software packages are also available for developing GIS applications.

GIS has wide applicability for water distribution system studies. Representation and analysis of water-related phenomena by GIS facilitates their management. By using geographic information from mapping, modeling, facilities management, and work order management, a drinking water distribution system manager can develop a detailed capital improvement program or operations and maintenance plan. Additional examples include (Schock and Clement 1995):

- GIS can assess the feasibility and impact of system expansion;

- GIS can provide the basis for investigating the occurrence of regulated contaminants for estimating the compliance cost or evaluating human health impacts;

- GIS can be used to investigate process changes for a water utility or to determine the effectiveness of some existing treatment such as corrosion control or chlorination; and

- GIS can assist in developing wellhead protection plans.

\subsection{Development of Hydraulic Models}

Shamsi $(1998,1999)$ provides a taxonomy to define the different ways a GIS can be linked to hydraulic models(Heaney et al., 1999). The three methods of developing GIS-based modeling applications defined by Shamsi are:

1. interchange method,

2. interface method, and

3. integration method

Figure 21.1 shows the differences among these methods. The interchange method employs a batch process approach to interchange (transfer) data between a GIS and a hydraulic model. In this method, there is no direct link between the GIS and the model. Both the GIS and the model are run separately and independently. The GIS database is analyzed to extract model input parameters, which are manually copied into a model input file. Similarly, model output data are manually copied into the GIS to create a new layer for presentation mapping purposes. Estimation of node demands from land use layers and determining node elevations from digital elevations models (DEM) are examples of the interchange method. 


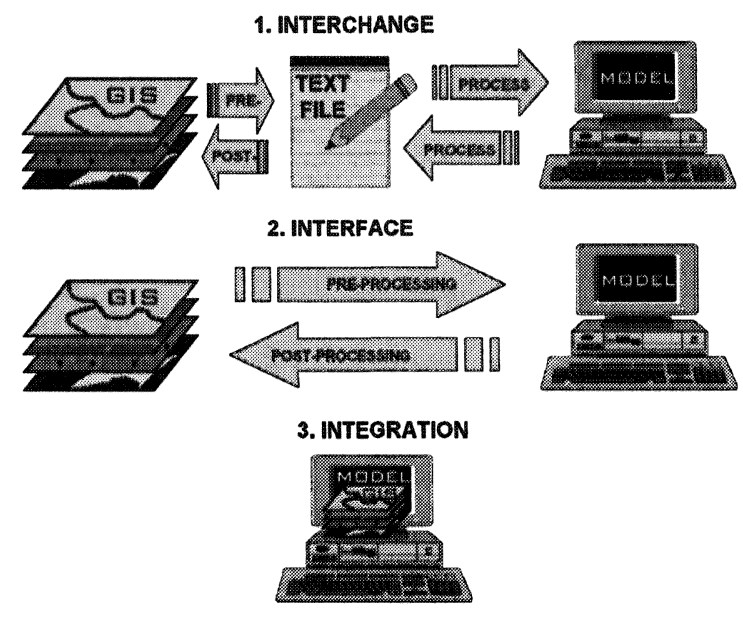

Figure 21.1 Three methods of GIS applications in computer modeling.

The interface method provides a direct link to transfer information between the GIS and the model. The interface method consists of at least the following two components: (i) a pre-processor, which analyzes and exports the GIS data to model input files; and (ii) a post-processor, which imports the model output and displays it as a GIS layer. The interface method basically automates the data interchange method. The automation is accomplished by adding modelspecific menus or buttons to the GIS software. In this method, options for data editing and launching the model from within the GIS are not available.

GIS integration, conversely, is a combination of a model and a GIS such that the combined program offers both the GIS and the modeling functions. This method represents the closest relationship between GIS and model.

\subsubsection{Model Integration Case Study}

This case study exemplifies application of the integration method. The integrated solution was developed by combining ArcView GIS software from Environmental Systems Research Institute (ESRI, Redlands, California, www.esri.com) and EPANET hydraulic modeling software from U.S. Environmental Protection Agency (EPA).

EPANET provides extensive capability to support decisions in the operation, management, planning and expansion of a water distribution system. A computer modeler who appreciates the popular phrase a picture is worth a 
thousand words should treasure the expression a map is worth a thousand numbers. GIS technology is an effective means of bridging the gap between the modeling information and its recipients. Unfortunately, many modelers do not have the technical expertise to develop, run, and maintain a complex GIS. ArcView is a user-friendly desktop mapping and GIS tool, which can be learned by most modelers without extensive training and experience. Due to its low cost, ease of use, and customization capability, ArcView was found to be suitable tool for developing the EPANET-GIS integration.

The EPANET Programmer's Toolkit is a dynamic link library (DLL) of functions that allow developers to customize EPANET's computational engine for their own specific needs. The functions can be incorporated into 32-bit Windows applications written in $\mathrm{C} / \mathrm{C}++$, Delphi Pascal, Visual Basic, or any other language that can call functions within a Windows DLL. There are over 50 functions that can be used to open a network description file, read and modify various network design and operating parameters, run multiple extended period simulations accessing results as they are generated or saving them to file, and write selected results to file in a user-specified format. The Toolkit should prove useful for developing specialized applications, such as optimization models or automated calibration models, that require running many network analyses as selected input parameters are iteratively modified. It also can simplify adding analysis capabilities to integrated network-modeling environments based on CAD, GIS, and database management packages (Rossman, 2000).

USFilter Engineering \& Construction (Pittsburgh, Pennsylvania) developed AVNET, a custom ArcView 3.x and EPANET 2.x integration. AVNET performs pre-processing of GIS data to create model input files. It also conducts post-processing of model results to create GIS maps of the model output results. Both the GIS and the modeling functions are available in ArcView. There is no need to exit ArcView to edit or run the model. Simply stated, AVNET provides seamless model input creation, model data editing, model execution, and model output display capabilities from within ArcView GIS. Other important features of AVNET are:

- use ArcView to modify the configuration of the water distribution system;

- compile new EPANET model input files reflecting those changes

- run the EPANET model from within ArcView; and

- use ArcView to map the water distribution system model and graphically display the results of the simulation on a georeferenced base map. 


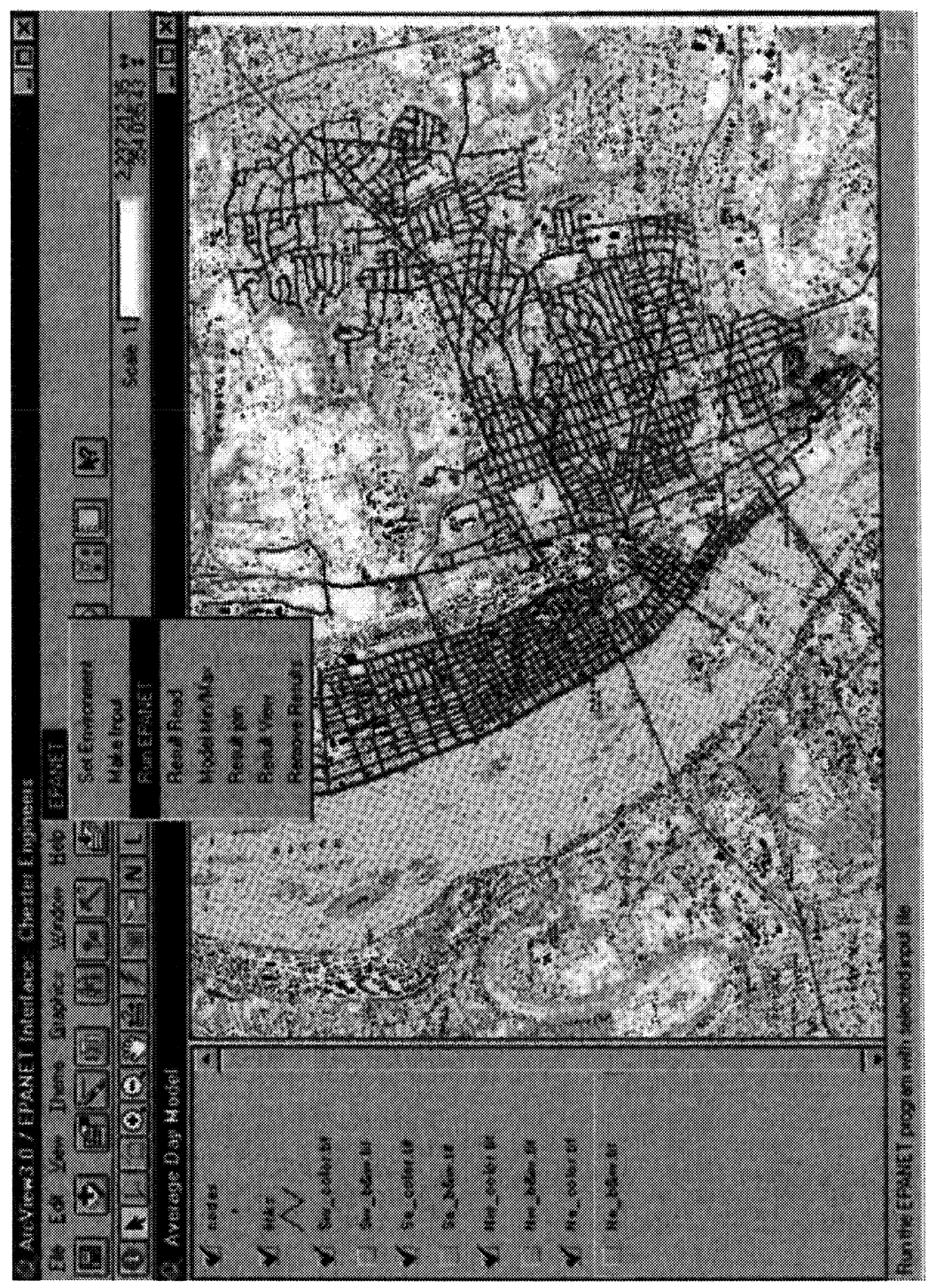

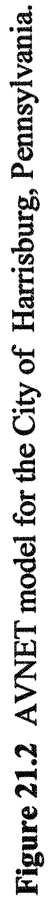


Figure 21.2 shows the AVNET model for the City of Harrisburg, Pennsylvania. This model consists of 2,500 pipes and 1,650 nodes, 4 tanks, and 6 pumps. Figure 21.3 shows EPANET output results presented as a thematic map in ArcView GIS. The first layer (drawn as dots) shows the minimum working pressures at the nodes. The second layer (drawn as lines) shows the maximum head losses for the pipes. These two layers can be overlayed to identify those areas where the pressures are low and head losses are high. Such areas usually represent inadequate pipe sizes for delivering the required flow rates and might be targeted for water main installation and replacement work.

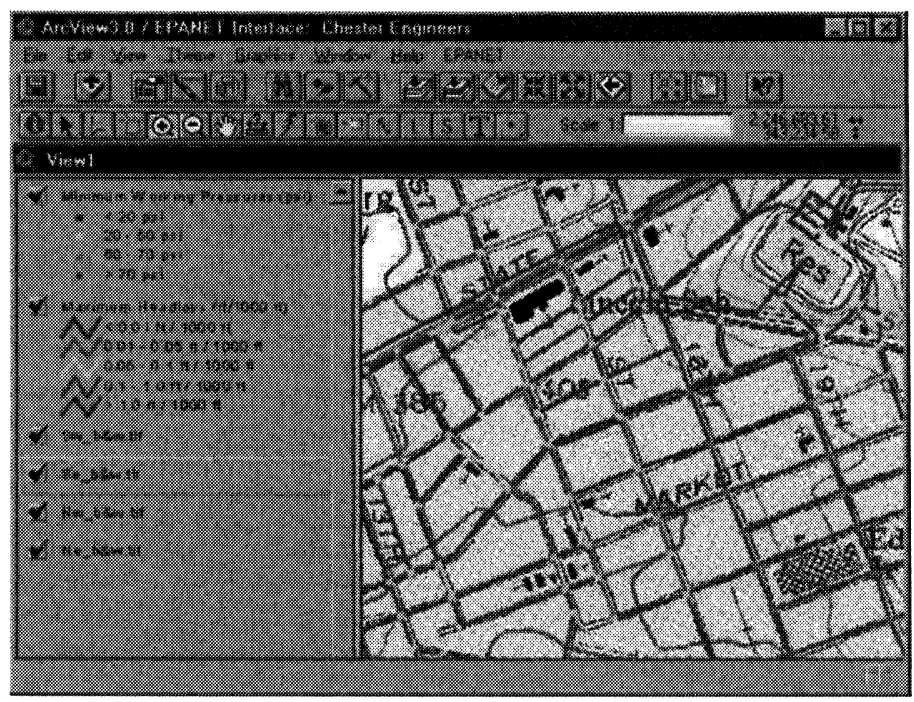

Figure 21.3 EPANET output results presented as a map in ArcView GIS.

\subsection{Network Skeletonization}

GIS and CAD layers of water distribution systems often have a level of detail that is unnecessary for analyzing system hydraulics. This can include hydrants, service connections, valves, fittings, and corner nodes. Although development of models that are an exact replica of the real system is now possible due to enormously powerful computers, the cost of purchasing, developing, and 
running very large models can be excessive. Attempting to include all the network elements could be a huge undertaking without a significant impact on the model results (Haestad et al., 2003). Modelers, therefore, resort to a network simplification process or "skeletonization."

Network simplification is needed to increase processing speed without compromising model accuracy. Manual skeletonization is a cumbersome process. Modelers, therefore, have resorted to rules-of-thumb, such as exclude all pipes smaller than a certain size. This approach requires a significant effort in locating and removing the candidate pipes.

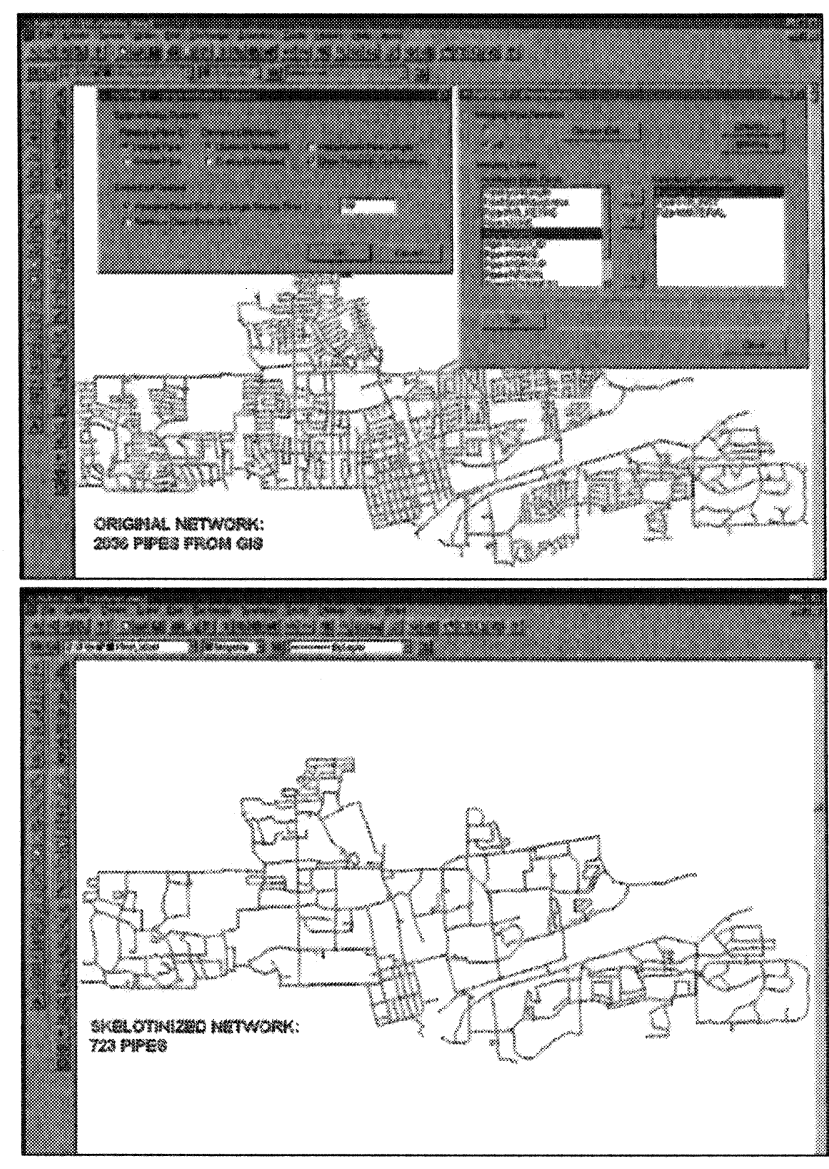

Figure 21.4 Skeletonizer's network simplification results. Top: original system with 2,036 pipes. Bottom: skeletonized network with 723 pipes (65\% reduction). (Maps courtesy of MWH Soft, Inc.) 
Automatic GIS-based skeletonization removes the complexity from the data, while maintaining the network integrity and hydraulic equivalency of the system. Reductions of $10-50 \%$ in the size of a network is possible. The Skeletonizer ${ }^{\mathrm{TM}}$ module of $\mathrm{H}_{2} \mathrm{OMap}$ water system modelingsoftware(MWH Soft, Inc., Pasadena, California, www.mwhsoft.com) and Skelebrator ${ }^{\mathrm{TM}}$ module of WaterCAD and WaterGEMS water system modeling software (Haestad Methods, Waterbury, Connecticut, www.haestad.com) are examples of tools that automate the skeletonization of CAD and GIS layers. Figure 21.4 shows an example of Skeletonizer skeletonization. The top screenshot shows the original network with 2,036 pipes imported from GIS. The bottom screenshot shows the skeletonized network with 723 pipes, indicating a $65 \%$ reduction in the size of the network.

\subsection{Estimation of Node Demands}

Node demand is an important input parameter of a hydraulic model representing the water consumption rate in units of volume / time (e.g. gpd or L/s). Demands are grouped by customer type (e.g. residential, commercial, industrial, or institutional) or magnitude (e.g. average daily, maximum daily, peak hourly, or fire flow). Manual estimation of node demands from customer billing records is a cumbersome process. Modelers, therefore, have traditionally resorted to approximate methods such as uniform distribution of the residential water use across all nodes followed by adding the nearest commercial and industrial use at various nodes. This approach requires a significant effort in fine tuning the node demands during the model calibration phase of the project.

GIS allows implementation of more accurate demand estimation methods. One such method utilizes the GIS to geocode (or geolocate) all individual water users (defined by the address at which each meter is located) and to assign the metered water consumption rates for each meter location to the nearest node in the model. The geocoding process links a street address to a geographic location and finds the coordinates of a point location based on its street address. This method assumes that a computer file (spreadsheet or delimited text file) containing meter addresses and metered water use for specified durations (e.g. quarterly or monthly) can be produced from the utility's billing database. If this information is available, the following procedure can be used to assign demands to nodes.

1. Create a GIS layer (e.g. a shapefile) representing all nodes in the model. 
2. Obtain a street centerline layer of the service area with address ranges. Such data can also be purchased from commercial GIS data vendors (Shamsi, 2002).

3. Obtain and pre-process customer meter location and consumption database.

4. Process the meter address and street address range data in GIS to geocode the meter locations into a point layer registered to model node layer.

5. Assign and aggregate individual meter demands to nearest nodes.

6. Analyze the assigned metered demand to determine unaccountedfor-water and metered demands that were not successfully geocoded. Uniformly distribute these remaining demands across nodes.

7. Load the node demand data into the hydraulic model.

The above procedure produces a highly accurate distribution of water use across the nodes. The intermediate product of point locations with associated demands can also be used to visualize the spatial distribution of individual demand points throughout the system. A metered demand database can also be created to include customer classes as defined in the billing system. This data can be used to assign differing demand patterns to residential, commercial, and industrial demand classes for use in extended period simulations.

Haestad Methods' WaterGEMS hydraulic modeling software has a Loadbuilder module that allows modelers to automatically calculate and assign demands to junctions using the following three methods (Haestad, 2002):

1. customer meter data method, if geocoded customer billing data are available;

2. water use data method, if billing meter data are not available; and

3. population / land use data method for forecasting future demands.

GIS can also use a census data layer (e.g. TIGER database from U.S. Bureau of Census, Shamsi, 2002) to determine population densities and residential and commercial customer distribution throughout the system.

\subsubsection{Demand Estimation Case Studies}

The City of Round Rock, Texas used Haestad Methods' WaterCAD hydraulic modeling software, ESRI's ArcView GIS package, and Microsoft's Access billing database for estimating the node demands. Approximately $95 \%$ of the City's 22,000 meters were assigned to precise geographic locations using 
geocoding. This approach provided accurate node demand data and reduced model calibration time (Haestad, 2002). The following steps were undertaken.

1. The geocoding process was performed in ArcView by linking the addresses within an existing parcels shapefile to the billing address in the water meter database.

2. Using WaterCAD's shapefile connection wizard, the node data from WaterCAD was synced to ArcView.

3. The modelers then drew polygons around the nodes and corresponding meters.

4. ArcView was used to sum the demand data for the meters in each polygon.

5. The total demand was applied to appropriate nodes.

6. The junction file with the newly calculated node demands was synchronized back to WaterCAD.

Pimpler and Zhan (2003) used a different method that worked well. They used a grid based (raster GIS) approach for estimating water demand for Lower Colorado River Authority that serves more than one million customers in Texas in an area spanning more than 29,800 square miles $\left(77,000 \mathrm{~km}^{2}\right)$. Their method involved four steps:

1. divide the service area into grid cells;

2. calculate a score for each grid cell based on weighted variables (e.g. land use, proximity to employment centers, etc.) considered important for water demand analysis;

3. distribute the service area population proportionally to each grid cell based on the score assigned to each cell; and

4. estimate water demand associated with each cell based on its allocated population.

\subsection{Estimation of Node Elevations}

Node elevation is also an important input parameter for a hydraulic model. Manual estimation of node elevations from topographic (contour) maps is a cumbersome process. GIS allows automatic computation of node elevations using the following procedure:

1. create a GIS layer (e.g. a shapefile) for the nodes in the model;

2. obtain a DEM layer covering the water distribution service area;

3. geo-reference (geo-register) the node and DEM layers (i.e.assign both layers the same map projection system). 
4. overlay the node layer over the DEM layer to assign DEM elevations to nodes; and

5. load the node elevation data into the model.

Figure 21.5 shows an overlay of a water distribution system layer over a DEM layer for calculating node elevations.

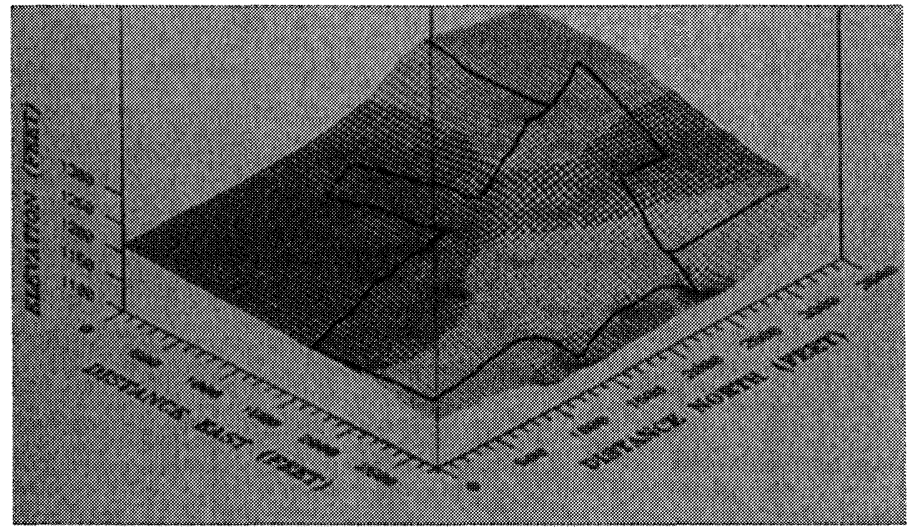

Figure 21.5 Water distribution pipes draped over a DEM.

\subsection{Water Main Isolation Trace}

Topology is defined as the spatial relationship between features. Spatial relationships between connecting or adjacent features, such as the pipes connected to a hydrant, which are so obvious to the human eye, must be explicitly defined to make the maps "intelligent." For example, our eyes can instantly identify the pipe serving a house just by looking at a paper map. A GIS, on the other hand, must mathematically compare the pipe and parcel layers to complete this task. Topology is, therefore, defined as a mathematical procedure for explicitly defining spatial relationships between features. The subject of topology is so sophisticated that it is considered a branch of mathematics (Shamsi, 2002). The GIS layers that have topology can be used to trace transportation or utility networks.

When a water main breaks, it should be isolated from the rest of the water distribution network for repairing the break or replacing the pipe. Water main isolation trace is defined as identifying those water distribution system valves 
that must be closed to repair or replace a broken water main. A water distribution system network layer that knows which links are connected to which nodes (i.e. has topology) can be used to traverse networks (Haestad et al., 2003) for solving the water main isolation problem. In addition to valve identification, GIS also allows an advance analysis of various water main shutdown scenarios, such as:

- refining the valve search based on inoperable valves,

- identifying dry pipes (i.e. pipes without water),

- identifying the affected customers served by dry pipes,

- identifying critical water service customers (e.g. hospitals) that cannot be interrupted,

- determining the extent of a service interruption to avoid unintentional shutdowns, and

- capturingleakhistory data useful in pipeline replacementmodeling.

\subsubsection{Isolation Trace Case Studies}

Figure 21.6 shows an isolation trace example from the PipeWorks example data. Pipeworks (now known as CityWorks) is an Automated Mapping / Facilities Management / GIS (AM/FM/GIS) software package from Azteca Systems (Sandy, Utah, www.azteca.com) that runs as an ArcView Extension. The trace is conducted by selecting the broken water main (midway on the pipe in the center of the map) and clicking on Isolation Trace button. The top screenshot shows that four valves must be closed to isolate the broken water main for repair or replacement. Next, it was assumed that one of these four valves located at the bottom of the screen is open inoperable, which would prevent this valve from being closed down. This scenario was modeled by changing the valve status from open operable to open inoperable. The actual valve status can be made available in the GIS database if field inspections data are integrated with the GIS database. The bottom screenshot shows that due to inoperable status of one of the valves, two additional (i.e., a total of six) valves must be closed.

In 2001, San Jose Water Company (SJWC) that services the City of San Jose, California, created an ArcView 8.1 application called Main Break to accomplish the above functions using Visual Basic and ArcObjects running against a personal geodatabase. The application was installed at SJWC's main office and on seven laptop computers. The laptop computers empowered the field personnel with a mobile GIS that allowed on-site identification of the valves to be closed (Coates, 2003). 


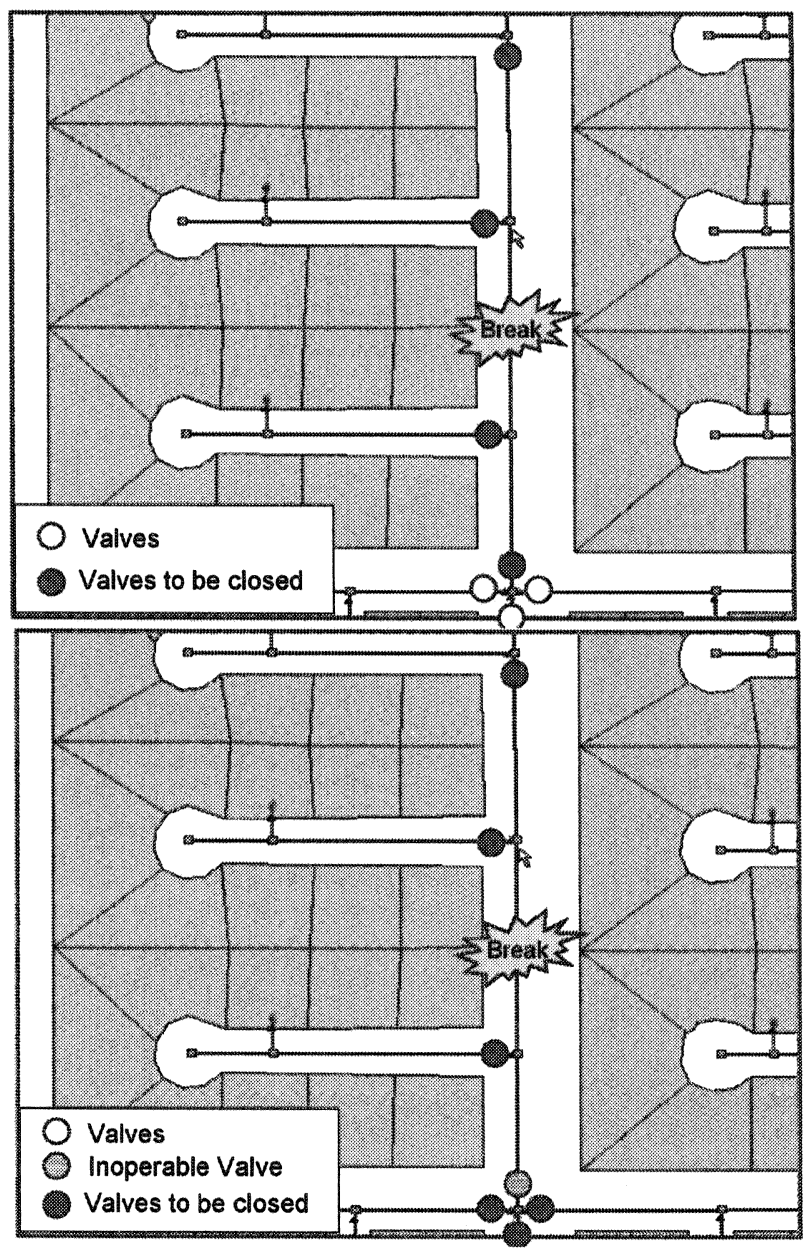

Figure 21.6 Isolation trace example. Above: all valves operable, Below: one valve inoperable

\subsection{Pressure Zone Trace}

GIS also allows identification of various pressure zones in a water distribution network. This is done by selecting a network pipe or node that is part of a larger pressure zone. GIS traces the water network in all possible directions until either sources of water or assets (e.g. pressure regulating valves - PRVs) that 
isolate water flow are found. Once the trace is complete, the user can assign a pressure zone attribute to the selected pipe or node.

Pidpa is a European company that produces and distributes drinking water to more than 1.1 million people throughout the Antwerp province in Belgium (Flanders). Pidpa used ESRI's ArcGIS 8.2 software and Miner and Miner's ArcFM 8.1.3 software to develop a data-centric GIS solution for the management of its drinking water network including functions for water main isolation trace and pressure zone trace (Horemans and Reynaert, 2003).

\subsection{Conclusions}

This chapter shows that GIS provides various useful applications for water distribution systems. The seven applications described in this chapter indicate that GIS can be used for various hydraulic modeling and system operation tasks. The case studies presented in the chapter illustrate that many water utilities are successfully using the GIS applications for modeling and operation of their water distribution systems. Various software examples presented in the chapter indicate that commercial-off-the-shelf software packages are also available for developing GIS applications.

\section{References}

Coates, J. (2003). "Water Main Shutdown Application Prevents Costly Mistakes." ArcUser, ESRI, January-March, 2003, Vol. 6, No. 1, 20-21.

Haestad Methods, (2002). "Water, A Demanding Solution." ClientCare Newsletter, Oct/Nov 2002, Haestad Press, Waterbury, Connecticut.

Haestad Methods, T. Walski, D. Chase, D. Savic, W. Grayman, S. Beckwith, and E. Koelle (2003). Advanced Water Distribution Modeling And Management, Haestad Press, Waterbury, Connecticut.

Heaney, J.P., Sample, D., and Wright, L. (1999). Geographical Information Systems, Decisions Support Systems, and Urban Stormwater Management. Cooperative Agreement Report No. CZ826256-01-0, U.S. Environmental Protection Agency, Edison, New Jersey.

Horemans, R. and B. Reynaert (2002). "ArcFM 8 Water : The Solution For The European Drinking Water Company." 17th ESRI European User Conference, Bruges, Belgium, October 14-16, 2002.

Pimpler, E. and B. Zhan (2003). "Drop by Drop, Modeling Water Demand on the Lower Colorado." Geospatial Solutions, Vol. 13, No. 5, May 2003, 38-41. 
Rossman, L.A.(2000). Computer Models /EPANET, Chapter 12 in Water Distribution Systems Handbook, L.W. Mays Editor, McGraw-Hill, 12.1-12.23.

Schock, M.R., and J.A. Clement (1995). "You Can't Do That With These Data! Or: Uses and Abuses of Tap Water Monitoring Analyses." Proceedings of the National Conference on Environmental Problem-Solving with Geographic Information Systems, EPA/625/R-95/004, Office of Research and Development, United States Environmental Protection Agency, Cincinnati, Ohio, September 1995, 3141.

Shamsi, U.M. 1998. "ArcView Applications in SWMM Modeling." Journal of Water Management Modeling R200-11. doi: 10.14796/JWMM.R200-11.

Shamsi, U.M. 1999. "GIS and Water Resources Modeling: State-of-the-Art." Journal of Water Management Modeling R204-05. doi: 10.14796/JWMM.R204-05. 